\title{
Independent and Dependent Contributions of Advanced Maternal and Paternal Ages to Autism Risk
}

\author{
Janie F Shelton ${ }^{1}$, Daniel J Tancredi ${ }^{3}$, and Irva Hertz-Picciotto ${ }^{1,2}$ \\ ${ }^{1}$ Department of Public Health Sciences, University of California, Davis \\ ${ }^{2}$ The UC Davis M.I.N.D. Institute, Sacramento, California \\ ${ }^{3}$ Department of Pediatrics and The Center for Healthcare Policy and Research, University of \\ California, Davis
}

\begin{abstract}
\section{LAY ABSTRACT}

Previous reports on autism among children born to older parents have yielded conflicting results as to which parent, or whether neither, or both, contributes to the risk. We analyzed ten years of births in California, comprising approximately 5 million children. Autism cases were identified from the California Department of Developmental Services database and linked to birth files from 1990-1999. Due to the size of this population, we were able to observe the trend in autism risk for each parent's age, restricted to a narrow age range of the other parent. Analysis was confined to singleton births with complete data on ages and educational levels of both parents $(n=4,947,935$, cases $=12,159)$. We observed consistent stepwise increased risk for autism with advancing maternal age regardless of the father's age, whereas increased risk with advancing paternal age was primarily observed among younger mothers, namely those $<30$ years of age. The different effects of father's age depending on the mother's age may indicate that the risk for autism from advancing maternal age past 30 years overwhelms the risk contributed by the father's age.

Additionally, we showed that if the distribution of mothers' age had been the only factor to change between 1990 and 1999, then we would have expected the cumulative incidence to have risen only 4.6\% during the decade from 1990 to 1999.
\end{abstract}

\section{SCIENTIFIC ABSTRACT}

Reports on autism and parental age have yielded conflicting results on whether mothers, fathers, or both, contribute to increased risk. We analyzed restricted strata of parental age in a ten-year California birth cohort to determine the independent or dependent effect from each parent. Autism cases from California Department of Developmental Services records were linked to State birth files (1990-1999). Only singleton births with complete data on parental age and education were included $(n=4,947,935$, cases $=12,159)$. In multivariate logistic regression models, advancing maternal age increased risk for autism monotonically regardless of the paternal age. Compared with mothers 25-29 years of age, the adjusted odds ratio (aOR) for mothers 40+ years was 1.51 (95\% CI: 1.35-1.70), or compared with mothers $<25$ years of age, aOR=1.77 (95\% CI, 1.56-2.00).

Corresponding author: Janie Shelton, Department of Public Health Sciences, MS1C, University of California Davis, CA 95616, USA, jfshelton@ucdavis.edu, Phone: (530) 752-7844. 
In contrast, autism risk was associated with advancing paternal age primarily among mothers $<30$ : aOR $=1.59$ (95\% CI, 1.37-1.85) comparing fathers 40+ vs. 25-29 years of age. However, among mothers > 30, the aOR was 1.13 (95\% CI, 1.01-1.27) for fathers 40+ vs. 25-29 years of age, almost identical to the aOR for fathers $<25$ years. Based on the first examination of heterogeneity in parental age effects, it appears that women's risk for delivering a child who develops autism increases throughout their reproductive years whereas father's age confers increased risk for autism when mothers are $<30$, but has little effect when mothers are past age 30 . We also calculated that the recent trend towards delayed childbearing contributed approximately a $4.6 \%$ increase in autism diagnoses in California over the decade.

\section{Keywords}

autism; maternal age; paternal age; effect measure modification; attributable risk; advanced maternal age; advanced paternal age; interaction

\section{INTRODUCTION}

Diagnoses of autism have increased in recent decades, and although controversy remains as to whether a true rise in incidence has occurred (Blaxill, 2004; Fombonne, 2005; Wing \& Potter, 2002), only a fraction of the 7-fold increase observed from 1990 through 2001 in California among five year olds can be explained by known factors such as changes to diagnostic criteria and a shift towards younger age at diagnosis (Hertz-Picciotto \& Delwiche, 2009). Autism is a pervasive developmental disorder (PDD) of deficits in social skills and communication, as well as repetitive and restricted behaviors occurring prior to age three (Diagnostic and Statistical Manual of Mental Disorders. Fourth Edition (DSMIV). 1994; International Classification of Diseases, Tenth Revision (ICD-10), 1994).

In parallel to the observed increase in autism cases, the number of births to women aged 4044 increased three-fold in California between 1982 and 2004 (Johnson, 2007), similar to the nationwide trend of advancing maternal age (CDC, 2004). Several studies conducted worldwide have reported advancing parental age as a risk factor for autism (Croen, Najjar, Fireman, \& Grether, 2007; Durkin et al., 2008; Larsson et al., 2005; Lauritsen, Pedersen, \& Mortensen, 2004; Reichenberg et al., 2006), yet the results remain inconsistent and the potential contribution of delayed childbearing to the increased incidence of autism has not been previously quantified.

In a 2007 California study of Kaiser Permanente members, Croen et al. (Croen et al., 2007) found an increased risk for autism spectrum disorders (ASD) per 10-year increment of advancing age in both mothers and fathers. In a 2006 historical study of Israeli military conscription records of a birth cohort of Jewish children from 6 consecutive years in the 1980's, Reichenberg et al. (Reichenberg et al., 2006) found an increased risk for ASD with increasing paternal age, but not maternal age, a measure which was missing on a large fraction of their sample. In 2005, Lauritsen et al. (Lauritsen, Pedersen, \& Mortensen, 2005) used categorical maternal and paternal age effects for autism (defined as childhood autism or atypical autism) --with the same reference category (25-29 y.o.) for each--and reported statistically significant increased adjusted relative risks for autism for each of the three 
highest paternal age categories (35-39 y.o., 40-44 y.o. and >44 y.o.) as well as the lowest maternal age category (12-19 y.o.) but none of the other maternal age categories in a Danish study population. In this analysis, parents' place of origin and psychiatric history were included as covariates. Another Danish study by Larsson et al. (Larsson et al., 2005) examining risk factors for infantile or atypical autism used similarly parameterized categorical maternal and paternal age effects and reported a set of adjusted odds-ratios from a preliminary model which showed that, compared to the reference age category of 25-29 y.o., the odds of autism were increased in children whose mothers were in the youngest $(<20$ y.o.) or whose fathers were in the highest age category ( $>39$ y.o.), after adjusting for perinatal risk factors. However, when additional covariates for parental psychiatric histories and socioeconomic status were included in these models, these estimated adjusted oddsratios were attenuated and were no longer statistically significant.

Contradicting both Reichenberg and Lauritsen, an Australian study by Glasson et al. (Glasson et al., 2004) reported statistically significantly increased adjusted relative risks for ASD and advancing maternal age in analyses that did not include paternal age because, according to the authors, "it did not emerge" as a significant predictor in preliminary stages of model building. An American study by Durkin et al. found an increased risk of ASD for maternal age 35 and over as compared to mothers $25-29$ (aOR=1.3, 95\% CI 1.1-1.6) and paternal age 40 and older compared to fathers 25-29 (1.4, 95\% CI 1.1-1.8) (Durkin et al., 2008). King et al. examined data from the California State Department of Developmental Services among children born between 1992 and 2000, and found an increased risk of autism for mothers $40+(1.8495 \%$ CI 1.37-2.47) and fathers 40+ (1.29 95\% CI 1.03-1.6), compared to parents under 30(King, Fountain, Dakhlallah, \& Bearman, 2009). Because autism is a rare condition, the majority of previous studies may have been underpowered for simultaneously estimating the separate effects of maternal and paternal ages, a problem further exacerbated by high correlation of these two exposures, which results in inflated variances for their estimated regression coefficients.

In this study, we evaluated the effect of advancing parental ages and the risk for autistic disorder in a cohort of 5 million births statewide in California between 1990 and 1999.

Through the application of stratified multivariate logistic regression modeling to an extremely large dataset, we were able to evaluate the independent and dependent effects of each parent's age within strata defined by narrow categories of the other parent's age. We thereby clarify how the impact of paternal age on child's autism risk depends on maternal age, a more complex relationship than has previously been understood. Additionally, we calculated the effect of the shift towards an older maternal age distribution on overall autism incidence rates for births between 1990 and 1999.

\section{METHODS}

To establish the cohort, we obtained an electronic birth record file for all births in the State of California between January 1, 1990 and December 31, 1999 including all available demographic data on the parents and child. To identify our case population, we obtained electronic files from the Department of Developmental Services (DDS) for all clients in the state of California born between January 1, 1990 and December 31, 1999. For children over 
the age of three, periodic evaluations are recorded on a Client Development and Evaluation Report (CDER), and for children under three, on an Early Start Report (ESR). Cases were defined by 1) an autism level of one (Full Syndrome Autism) on any CDER record, 2) an ICD code of 299.0 (Autistic Disorder), or 3) a checkmark for "Autism" under Developmental Disabilities on the ESR. The diagnosis was recorded for the date of the earliest CDER or ESR file. The DDS case file and the birth record file were merged to create the analysis file.

\section{COHORT EXCLUSIONS}

Because diagnosis data were only available through the end of 2006, to ensure comparability of rates among the 10 adjacent years of birth cohorts in our study, we included only cases diagnosed prior to age 6 (case $\mathrm{N}=13,733$ out of a population of 5,639,867 births). In this manner, each birth year from 1990 to 1999 had equivalent time at risk of diagnosis. Because previous key studies adjusted for multiplicity, we restricted to singletons to enhance comparability, which removed 566 cases and 134,050 controls. Five cases and 2,069 controls were excluded due to missing information for mother's age and 669 cases and 382,250 controls were excluded due to missing information for father's age. Sixteen cases and 9,013 controls were removed due to missing information on maternal education, and 308 cases and 159,922 controls were removed due to missing information on father's education, an important proxy for socio-economic status. The potential for bias due to the amount of missing information on maternal and paternal age and education was evaluated using multiple imputation (under the assumption that data were missing at random) and sensitivity analysis (to test plausible departures from the non-informative missingness assumption). Ten cases and 3,053 controls were excluded due to improbably high parity (over 20 prior births). Altogether, these exclusions reduced the sample size by $12.3 \%$ (11.5\% among cases, and $12.3 \%$ among controls). Table 1 describes the demographics of the population with complete information on both parent's age and education. In the regression analyses, a special category "missing" was added to each of the parental race/ethnicity regressor classifications in order to retain observations with incomplete information on these two variables. Comparisons between models with adjustment for missingness of race/ ethnicity vs. exclusion of those in the missing category showed that bias in the point estimate was no more than $3 \%$ in any one parental age category. A total of 12,159 cases and 4,935,776 controls described in Table 1 had complete information on all dependent and independent variables to be specified in regression analyses and were used in all subsequent analysis. Secondary analyses were conducted on multiple births only and gender stratified births.

\section{MODEL SELECTION}

The net effects of maternal and paternal age on the risk of autism after adjustment for potential confounders were modeled by logistic regression, with the parental age terms specified either as continuous or categorical effects. For categorical specifications, parental age was stratified into five categories: $<25,25-29,30-34,35-39$, and $>40$. The cohort median age was $27(\mathrm{SD}=6.2)$ for mothers and $29(\mathrm{SD}=7.0)$ for fathers; correspondingly, the 
large 25 to 29 y.o. age group was selected as the reference category for both parental age effects.

Covariates were included in the final regression model: if they enhanced comparability with previous studies; if they were identified as confounders in our a priori specification of plausible directed acyclic graphs representing causal relationships among study variables; and/or if their addition to preliminary models resulted in relative changes in the parental age regression coefficients of greater than $10 \%$. Covariates initially considered in the study were gestational diabetes, pre-eclampsia, parental race/ethnicity, parental education, year of birth, and parity. Of the independent variables tested for inclusion in the final model, parental education demonstrated the largest confounding effect. The final covariates selected for adjustment in all models were: parental education (the sum of both parents' years of education), year of child's birth, race/ethnicity of mother, race/ethnicity of father, mother's parity, and insurance payment type (public, private, other). All analyses were conducted using SAS statistical software version 9.1 (SAS Institute, Cary, NC).

\section{AGE-STRATIFIED ANALYSIS \& HETEROGENEITY}

Due to the strong correlation between maternal and paternal age $(\rho=0.74, p<0.0001)$, which could decrease the precision of inferences for these effects in a full-dataset analysis, adjusted odds ratios for the effects of advancing paternal (maternal) age were estimated using stratified multivariate logistic regression in strata defined by narrow (five-year) maternal (paternal) age groups. This stratified analysis allowed us to assess, for example, the effect of increasing paternal age in strata in which maternal age was restricted to a narrow age range (e.g. 30 to 34 years). The smallest maternalXpaternal age cell included in this analysis was for father's 25- 29 and mothers $40+$ (case $\mathrm{N}=11$, control $\mathrm{N}=4,066$ ). The cell for fathers $<25$ and mothers $40+$ was suppressed due to a case $\mathrm{N}$ of 3 .

Based on results from the restricted age models, we subsequently fit a model to the full dataset that included all ages to provide an overall assessment of effect modification of paternal age by maternal age. In addition to the 5-level maternal and paternal age terms and other covariates, this model included an interaction term comprised of the 5-level paternal age categorical effect multiplied by the binary indicator for maternal age $(1=30$ and under, $0=$ over 30).

\section{CALCULATION OF INCREASE IN INCIDENT CASES OF AUTISM DUE TO ADVANCING MATERNAL AGE}

To evaluate the effect of advancing maternal age on the incidence of autism, we applied the maternal age distribution of 1999 to risks for autism at each maternal age in the 1990 birth cohort. We thereby created a pseudo population for 1990 having the age structure of 1999 births and all other factors held constant, and we used this to estimate the projected number of extra cases. This excess was expressed as a percentage of the autism incidence in 1990 births to demonstrate the potential impact of changes in maternal age over the decade. 


\section{VARIANCE INFLATION FACTOR ADJUSTMENT}

Clustering effects shared by births from the same mother could affect the precision of point and variance estimates of regression coefficients and the resulting inferences. However, the analysis dataset did not allow us to link across records from the same mother, preventing the straightforward use of clustered data regression procedures to account for these effects. Therefore, we applied a post-hoc procedure, using conservative assumptions to adjust for these biases, based on the standard formula for the variance inflation factor (VIF, or design effect) associated with clustered data, $\mathrm{VIF}=1+(\mathrm{m}-1) \mathrm{R}$, where $\mathrm{m}$ is the average cluster size and $\mathrm{R}$ is the within-cluster correlation. Specifying that the within-mother correlation for the outcome indicator would likely be no higher than $10 \%$, given that the risk for autism among full siblings of cases is less than 10\% (Muhle, Trentacoste, \& Rapin, 2004), and that the average cluster size would be less than 3.0, given an estimated median parity of 2 prior births per mother, we computed that a factor of 1.21 would be a reasonable yet conservative (over) estimate of the variance inflation due to clustering. The square root of 1.21 was then used to multiply the standard error of the parameter estimate when computing $95 \%$ confidence intervals for logistic regression coefficients, resulting in an expansion of these confidence intervals by $10 \%$. The $95 \%$ confidence intervals for the adjusted odds ratios corresponding to these regression coefficients were computed by exponentiating the endpoints of the expanded confidence intervals. Given that the reported 95\% CIs reflect a conservative (over) adjustment for clustering, they are likely to have higher than nominal coverage probabilities for parameters of interest, while our actual type-1 error rates for hypothesis tests are likely to be lower than the nominal value of $5 \%$.

\section{RESULTS}

Children with autism are more likely than controls to be male (males: females $=4.6: 1$ ), to have older parents, and to be either non-Hispanic white or Asian as compared to children without autism (Table 1). The median age of mothers at the time of delivery was 30 for cases and 27 for controls. The median age of fathers was 32 among cases and 29 among controls.

\section{Maternal Age}

Modeled as a continuous variable, maternal age showed an approximate eighteen percent increase in the risk of autism per five -year increment in age (adjusted odds ratio (OR) 1.18, 95\% CI: 1.04-1.33). When maternal age was modeled categorically, a stepwise increase in risk for autism per 5-year interval of age. Adjusted models showed that, compared with mothers $25-29$ years of age, mothers over age 40 had $51 \%$ higher odds of having a child with autism (aOR 1.51, 95\% CI: 1.35-1.70). When compared with mothers <25 years, the adjusted OR was 1.77 (95\% CI 1.56-2.00). The maternal age effect was slightly stronger among female children and much stronger among children from multiple births (see Table 2) increased risk from advancing paternal age, nor evidence of a trend (Fig. 1B).

In analyses examining the effect of mother's age within restricted subsets of father's age, mother's age showed a similar pattern across all categories of paternal age. In all subsets except for fathers over age 40 , mothers $<25$ show a protective effect followed by stepwise 
increases in risk with advancing age. The highest risk relative to the reference group (mothers 25-29) was observed among fathers < 25 and mothers $35-39$ (aOR 2.09, 95\% CI 1.27-3.43) (Figure 1a).

\section{Paternal age}

When father's age was modeled continuously, each five-year increase in age resulted in an approximate eleven percent increase in the odds for autism (aOR 1.11, 95\% CI 0.98-1.24). Categorical modeling showed fathers $>40$ had $36 \%$ increased odds of having a child with autism compared to fathers aged 25 - 29 (aOR 1.36, 95\% CI 1.26-1.47), or 78\% when compared with fathers aged $<25$ years (aOR $1.7895 \%$ CI 1.62-1.97). The paternal age effect was stronger in multiple births than among the singletons, and has a slight inverse relationship with the male to female (M: F) ratio among cases (Table 2).

The effect of father's age within restricted subsets of mother's age reflected a dose-response trend among mothers under 30, whereas for mothers over age 30, the pattern of autism risk with paternal age showed no clear monotonic trend (Figure 1b). Among mothers under age 30 , children with a young father $(<25)$ are protected and there is a stepwise increase per fiveyear increment of father's age. For example, among mothers $<25$, fathers $40+$ were twice as likely (aOR 1.91, 95\% CI 1.32-2.74) to have a child with autism as compared to fathers aged 25-29. Among mothers 30-34, 35-39 and 40+, the restricted analysis show no statistically significant increased risk of paternal age (Figure 1b).

\section{Effect measure modification}

Because of the heterogeneity of effect observed in Graph 1b, interaction terms were tested in the full model to evaluate effect measure modification of paternal age by maternal age. Among mothers under 30, very young fathers (<25 y.o.) show a $24 \%$ decreased risk as compared to the reference group of fathers 25-29 (aOR $0.7695 \%$ CI 0.70-0.82), whereas older fathers (40+) show a $60 \%$ elevated risk (aOR $1.5995 \%$ CI 1.37-1.85). Among mothers 30 and older, only fathers in the 40+ age category showed any effect of increased risk, contributing a $13 \%$ increased risk of autism (aOR 1.13 1.01-1.27). All other effect estimates of paternal age among mothers $30+$ were not statistically significant (Table 3 ).

\section{Attributable fraction of cases due to increasing maternal age}

In 1990,679 of the total 540,004 births were to mothers 40 or older. In 1999, 1,831 of the 453,617 births were to mothers 40 and older, a 3.2 fold increase in the proportion of births to mothers in that age group over a ten-year period. We estimated what the expected number of autism cases would be if the older maternal age distribution of the 1999 birth cohort were applied to the age-specific autism risks from the 1990 birth cohort, and found an excess of 45.6 cases would have occurred. This result indicates a $4.6 \%$ increase in incidence solely attributable to the shift towards older maternal age between 1990 and 1999 (Table 4).

\section{Stratified Models}

Among boys, the risk of autism and parental age is nearly identical to the adjusted analysis combining both sexes due to the overrepresentation of boys among affected children. The increased risk, for older mothers, of having a daughter who develops autism is slightly 
stronger, but not statistically significantly so. The trend of increased risk with mothers of 40 or over is stronger among multiple births ( $\mathrm{aOR}=2.0295 \% \mathrm{CI} 1.29-3.16)$ than singletons $(\mathrm{aOR}=1.5195 \% \mathrm{CI} 1.35-1.70)$, but they are not statistically significantly different for any of the parental age strata. The male to female ratio was reported by age strata, and decreases slightly as fathers age, but not in a monotonic fashion (Table 2).

\section{DISCUSSION}

We demonstrate that advancing maternal age increases the risk of autism independent of father's age, while advancing father's age increases the risk of autism primarily for mothers under 30. Among mothers over 30, we observe a small increased risk among fathers $40+$. The strength of this study was the ability to examine the effect of one parent's increasing age within a narrow interval of the other parent's age. Due to the low incidence of autism, obtaining sufficient numbers of cases among categories of discordant age pairings at the extreme upper age limit of parental age requires a rather large cohort, which the majority of previous studies, did not have available. In general, a very large study population is needed to disentangle the effects of two highly associated variables, maternal and paternal age.

Our findings initially appear to contradict a study by Reichenberg and colleagues in Israel (Reichenberg et al., 2006), which received widespread publicity implicating father's age as a considerable risk factor for autism, $(\mathrm{aOR}=5.75,95 \%$ CI $2.65-12.46$ for fathers 40 to 49 as compared to fathers 15 to 29). Upon replication of the categories of age used in the analyses of the Israeli population, we observed an adjusted odds ratio of 1.38 (95\% CI 1.22-1.41) for fathers 40 to 49 as compared to fathers 15 to 29 in the California cohort, adjusted for maternal age, education of both parents, race/ethnicity of both parents, year of birth, payment type, and parity. A major difference between the two studies is the proportion of older mothers. The California cohort had 113,080 mothers over age 40, of which 501 were case mothers, whereas the Israeli cohort had only 588 mothers with 4 cases in that age category. First, inferences based on a cell size of less than 5 are problematic as random error may play a large role in the findings. Second, the older maternal age distribution of the California cohort (2.3\% of mothers 40 and older compared with $0.4 \%$ in the Israeli cohort) permitted a more robust statistical analysis of paternal age within maternal age strata. Thus, in addition to random error, because the Israeli cohort of mothers was younger, older fathers paired primarily with younger mothers may have contributed to the large paternal age effect observed in that study. In our much larger cohort, the aOR for older paternal age in younger mothers did not exceed 2.0.

Prior to comparison with other studies, it should be noted that some studies examined all ASDs while others used a more restrictive case group of Autism (Autistic Disorder) alone, and since the former includes Asperger's Syndrome and Pervasive Developmental Delay Not Otherwise Specified, one might expect some differences in the impact of parental ages. Nevertheless, although they are behaviorally distinct, they may or may not be etiologically distinct. The majority of studies examine risk factors for all ASDs due to low sample sizes of Autistic Disorder, which may dilute any observed effect if they are etiologically different with regard to parental age. On the other hand, to the extent these distinctions represent functionally different degrees of impairment on a continuous scale of behavioral 
abnormalities, we cannot say for sure that studies examining ASD and Autistic Disorder are incomparable.

We considered the possibility of bias resulting from missing information on father's age from the birth records (approximately 7\% for cases and 9.6\% of non-cases). We conducted a multiple imputation and a sensitivity analysis. SAS PROC MI was used to generate 5 multiply imputed datasets, imputing values for missing variables using sequential regression models beginning with the variable having the fewest missing observations: maternal age, followed by maternal education, paternal age, and finally paternal education. The regression models used for imputation included all covariates from the fully adjusted model, as well as case status. Logistic regression models for each of the multiply imputed datasets were fit for mothers $<30$ years of age and $\geq 30$ years of age, with the point and variance estimates then combined by SAS PROC MIANALYZE. Comparison of these results with the completecase analysis reported here showed no more than a 3\% difference in the ORs for any category of paternal age, indicating that our results are robust to the absence of data on these four variables under the assumption that missingness was not informative. To test this latter assumption, we also conducted a sensitivity analysis to estimate potential bias induced if the missing fathers' ages were jointly dependent on maternal age and case status; the original trend associated with paternal age among younger mothers and lack of trend among older mothers remained.

Increased maternal age is an established risk factor for infertility, early fetal loss, chromosomal aberrations, increased copy number variations, low birth weight, and congenital malformations (Berkowitz, Skovron, Lapinski, \& Berkowitz, 1990; Martin, 2008). More recently, advanced paternal age has been associated with poor birth outcomes and has been shown to increase the risk of schizophrenia (Brown et al., 2002), neurocognitive deficits (Saha et al., 2009), childhood cancer (Dockerty, Draper, Vincent, Rowan, \& Bunch, 2001), low birth weight (Reichman \& Teitler, 2006), pre-eclampsia (Harlap et al., 2002), and miscarriage related to trisomic spontaneous abortion (Nybo Andersen, Hansen, Andersen, \& Davey Smith, 2004). Additionally, older paternal age has also been associated with point mutations in the RET gene, FGFR 2 gene, and FGFR 3 genes as well as generalized DNA damage, and shortened telomeres (Sartorius \& Nieschlag, 2009). Although poor birth outcomes have been associated with advanced age, the specific mechanisms are not well understood. Genetic, epigenetic, immunologic, endocrine, environmental, and other factors may underlie the increased risks for autism associated with parental aging.

In addition to the overall systemic changes that occur as a parent ages, epigenetic changes over time may enable an older parent to transfer a multitude of molecular functional alterations to a child. As a person ages, post-transcriptional histone modifications and methylation patterns are influenced by environmental exposures independent of one's genetic sequence (Fraga \& Esteller, 2007). Those acquired changes can then be encoded within the DNA and dictate gene expression patterns in subsequent generations (Tucker et al., 1996). Thus, epigenetics may be involved in the risk contributed by advancing parental age as a result of changes induced by stresses from environmental chemicals, co-morbidity, or assisted reproductive therapy. 
The Centers for Disease Control and Prevention report that use of assisted reproductive technology has more than doubled between 1996 and 2005 (Wright, Chang, Jeng, \& Macaluso, 2008). Older parents are more likely than younger ones to make use of these technologies (Sunderam et al., 2009). A few studies have evaluated neurodevelopment in children born from intracytoplasmic sperm injection and in vitro fertilization, and none have demonstrated significant increased risk for autism or autism spectrum disorders, yet the majority of studies have only followed up through the period of infancy (Middelburg, Heineman, Bos, \& Hadders-Algra, 2008). However, mothers who become pregnant after 35 (naturally or through ART) are already at increased risk of complications of pregnancy, labor, and delivery such as vaginal bleeding, prolonged labor, prematurity (Brimacombe, Ming, \& Lamendola, 2007), breech position, and low APGAR score at 5 minutes(Larsson et al., 2005), factors that have also been associated with autism.

Another avenue by which age may be affecting autism risk is through maternal autoimmunity. In 2008, a study comparing 61 mothers of children with autistic disorder to 102 controls found that $11.5 \%$ of mothers of autistic children (7/61) had antibodies to fetal brain protein compared to 0 mothers in the control group (Braunschweig et al., 2008).

Additionally, advancing age has been associated with an increase, advancing age has been associated with an increase in autoantibody production (Larbi, Fulop, \& Pawelec, 2008).

Finally, environmental exposures such as polychlorinated biphenyls (PCBs), polybrominated diphenyl ethers (PBDEs), heavy metals, pesticides, and plasticizers can either bioaccumulate or have a cumulative effect of exposure and all have been hypothesized to play a role in autism etiology (Hertz-Picciotto et al., 2006). It is plausible that multiple exposure types may increase the risk of autism through a common pathway or pathways (i.e. mitochondrial function, thyroid function, epigenetics, hormonal alterations) and be represented as a generalized increased risk with age. In this case, maternal or paternal age would serve as an index of lifetime exposure status and be a proxy for the true underlying etiologic agent.

\section{SUMMARY}

These data show that the risk of having a child with full syndrome autism increases with maternal age, but increased risk from advancing paternal age primarily occurs among younger mothers $(<30)$. These findings suggest the increased risk associated with older fathers is overwhelmed by the maternal age effect in the later years of fertility.

Alternatively, these findings may suggest a different mechanism for paternally- vs. maternally- mediated age effects. We calculated that the effect of advancing maternal age on the overall incidence of autism is apparent, yet small (4.6\%) in comparison to a several hundred percent increase throughout the period of this study (Hertz-Picciotto \& Delwiche, 2009). Future studies are needed to explore social and biological explanations for the relationship between parental age and autism.

\section{Acknowledgments}

We would like to acknowledge Jasmine Nettiksimmons for her generous assistance with the visualization of our data in Figures 1a, 1b. Special thanks to Lora Delwiche for her work preparing this dataset. This work was 
supported by grants from NIEHS (P01-11269, R01-015359), and the U.S. EPA (STAR ]R-829388 \& R833292) and the UC Davis School of Medicine and Office of Graduate Studies.

\section{LITERATURE CITED}

Berkowitz GS, Skovron ML, Lapinski RH, Berkowitz RL. Delayed childbearing and the outcome of pregnancy. N Engl J Med. 1990; 322(10):659-664. [PubMed: 2304491]

Blaxill MF. What's going on? The question of time trends in autism. Public Health Rep. 2004; 119(6): 536-551. [PubMed: 15504445]

Braunschweig D, Ashwood P, Krakowiak P, Hertz-Picciotto I, Hansen R, Croen LA, et al. Autism: maternally derived antibodies specific for fetal brain proteins. Neurotoxicology. 2008; 29(2):226231. [PubMed: 18078998]

Brimacombe M, Ming X, Lamendola M. Prenatal and birth complications in autism. Matern Child Health J. 2007; 11(1):73-79. [PubMed: 17053965]

Brown AS, Schaefer CA, Wyatt RJ, Begg MD, Goetz R, Bresnahan MA, et al. Paternal age and risk of schizophrenia in adult offspring. Am J Psychiatry. 2002; 159(9):1528-1533. [PubMed: 12202273]

CDC. National Vital Statistics Reports. 2004. o. Document Number)

Croen LA, Najjar DV, Fireman B, Grether JK. Maternal and paternal age and risk of autism spectrum disorders. Arch Pediatr Adolesc Med. 2007; 161(4):334-340. [PubMed: 17404129]

Diagnostic and Statistical Manual of Mental Disorders. Fourth Edition (DSM-IV). Washington, DC: American Psychiaric Association; 1994.

Dockerty JD, Draper G, Vincent T, Rowan SD, Bunch KJ. Case-control study of parental age, parity and socioeconomic level in relation to childhood cancers. Int J Epidemiol. 2001; 30(6):1428-1437. [PubMed: 11821358]

Durkin MS, Maenner MJ, Newschaffer CJ, Lee LC, Cunniff CM, Daniels JL, et al. Advanced Parental Age and the Risk of Autism Spectrum Disorder. Am J Epidemiol. 2008

Fombonne, E. Epidemiological studies of Pervasive Developmental Disorders. In: P.R. Vokmar, FR.; Klin, A.; Cohen, D., editors. Handbook of Autism and Pervasive Developmental Disorders. 3 ed.. Vol. Vol. 1. Hoboken, NJ: Wiley; 2005. p. 42-69.

Fraga MF, Esteller M. Epigenetics and aging: the targets and the marks. Trends Genet. 2007; 23(8): 413-418. [PubMed: 17559965]

Glasson EJ, Bower C, Petterson B, de Klerk N, Chaney G, Hallmayer JF. Perinatal factors and the development of autism: a population study. Arch Gen Psychiatry. 2004; 61(6):618-627. [PubMed: 15184241]

Harlap S, Paltiel O, Deutsch L, Knaanie A, Masalha S, Tiram E, et al. Paternal age and preeclampsia. Epidemiology. 2002; 13(6):660-667. [PubMed: 12410007]

Hertz-Picciotto I, Croen LA, Hansen R, Jones CR, van de Water J, Pessah IN. The CHARGE study: an epidemiologic investigation of genetic and environmental factors contributing to autism. Environ Health Perspect. 2006; 114(7):1119-1125. [PubMed: 16835068]

Hertz-Picciotto I, Delwiche L. The Rise in Autism and the Role of Age at Diagnosis. Epidemiology. 2009; 20(1):84-90. [PubMed: 19234401]

International Classification of Diseases, Tenth Revision (ICD-10). Geneva: World Health Organization; 1994.

Johnson, HP. Birth Rates in California. Public Policy Institute of Californiao; 2007. Document Number)

King MD, Fountain C, Dakhlallah D, Bearman PS. Estimated autism risk and older reproductive age. Am J Public Health. 2009; 99(9):1673-1679. [PubMed: 19608957]

Larbi A, Fulop T, Pawelec G. Immune receptor signaling, aging and autoimmunity. Adv Exp Med Biol. 2008; 640:312-324. [PubMed: 19065799]

Larsson HJ, Eaton WW, Madsen KM, Vestergaard M, Olesen AV, Agerbo E, et al. Risk factors for autism: perinatal factors, parental psychiatric history, and socioeconomic status. Am J Epidemiol. 2005; 161(10):916-925. discussion 926-918. [PubMed: 15870155] 
Lauritsen MB, Pedersen CB, Mortensen PB. The incidence and prevalence of pervasive developmental disorders: a Danish population-based study. Psychol Med. 2004; 34(7):1339-1346. [PubMed: 15697060]

Lauritsen MB, Pedersen CB, Mortensen PB. Effects of familial risk factors and place of birth on the risk of autism: a nationwide register-based study. J Child Psychol Psychiatry. 2005; 46(9):963971. [PubMed: 16108999]

Martin RH. Meiotic errors in human oogenesis and spermatogenesis. Reprod Biomed Online. 2008; 16(4):523-531. [PubMed: 18413061]

Middelburg KJ, Heineman MJ, Bos AF, Hadders-Algra M. Neuromotor, cognitive, language and behavioural outcome in children born following IVF or ICSI-a systematic review. Hum Reprod Update. 2008; 14(3):219-231. [PubMed: 18367619]

Muhle R, Trentacoste SV, Rapin I. The genetics of autism. Pediatrics. 2004; 113(5):e472-e486. [PubMed: 15121991]

Nybo Andersen AM, Hansen KD, Andersen PK, Davey Smith G. Advanced paternal age and risk of fetal death: a cohort study. Am J Epidemiol. 2004; 160(12):1214-1222. [PubMed: 15583374]

Reichenberg A, Gross R, Weiser M, Bresnahan M, Silverman J, Harlap S, et al. Advancing paternal age and autism. Arch Gen Psychiatry. 2006; 63(9):1026-1032. [PubMed: 16953005]

Reichman NE, Teitler JO. Paternal age as a risk factor for low birthweight. Am J Public Health. 2006; 96(5):862-866. [PubMed: 16571696]

Saha S, Barnett AG, Foldi C, Burne TH, Eyles DW, Buka SL, et al. Advanced Paternal Age Is Associated with Impaired Neurocognitive Outcomes during Infancy and Childhood. PLoS Med. 2009; 6(3):e40. [PubMed: 19278291]

Sartorius GA, Nieschlag E. Paternal age and reproduction. Hum Reprod Update. 2009

Sunderam S, Chang J, Flowers L, Kulkarni A, Sentelle G, Jeng G, et al. Assisted reproductive technology surveillance--United States, 2006. MMWR Surveill Summ. 2009; 58(5):1-25. [PubMed: 19521336]

Tucker KL, Beard C, Dausmann J, Jackson-Grusby L, Laird PW, Lei H, et al. Germ-line passage is required for establishment of methylation and expression patterns of imprinted but not of nonimprinted genes. Genes Dev. 1996; 10(8):1008-1020. [PubMed: 8608936]

Wing L, Potter D. The epidemiology of autistic spectrum disorders: is the prevalence rising? Ment Retard Dev Disabil Res Rev. 2002; 8(3):151-161. [PubMed: 12216059]

Wright VC, Chang J, Jeng G, Macaluso M. Assisted reproductive technology surveillance--United States, 2005. MMWR Surveill Summ. 2008; 57(5):1-23. [PubMed: 18566567] 

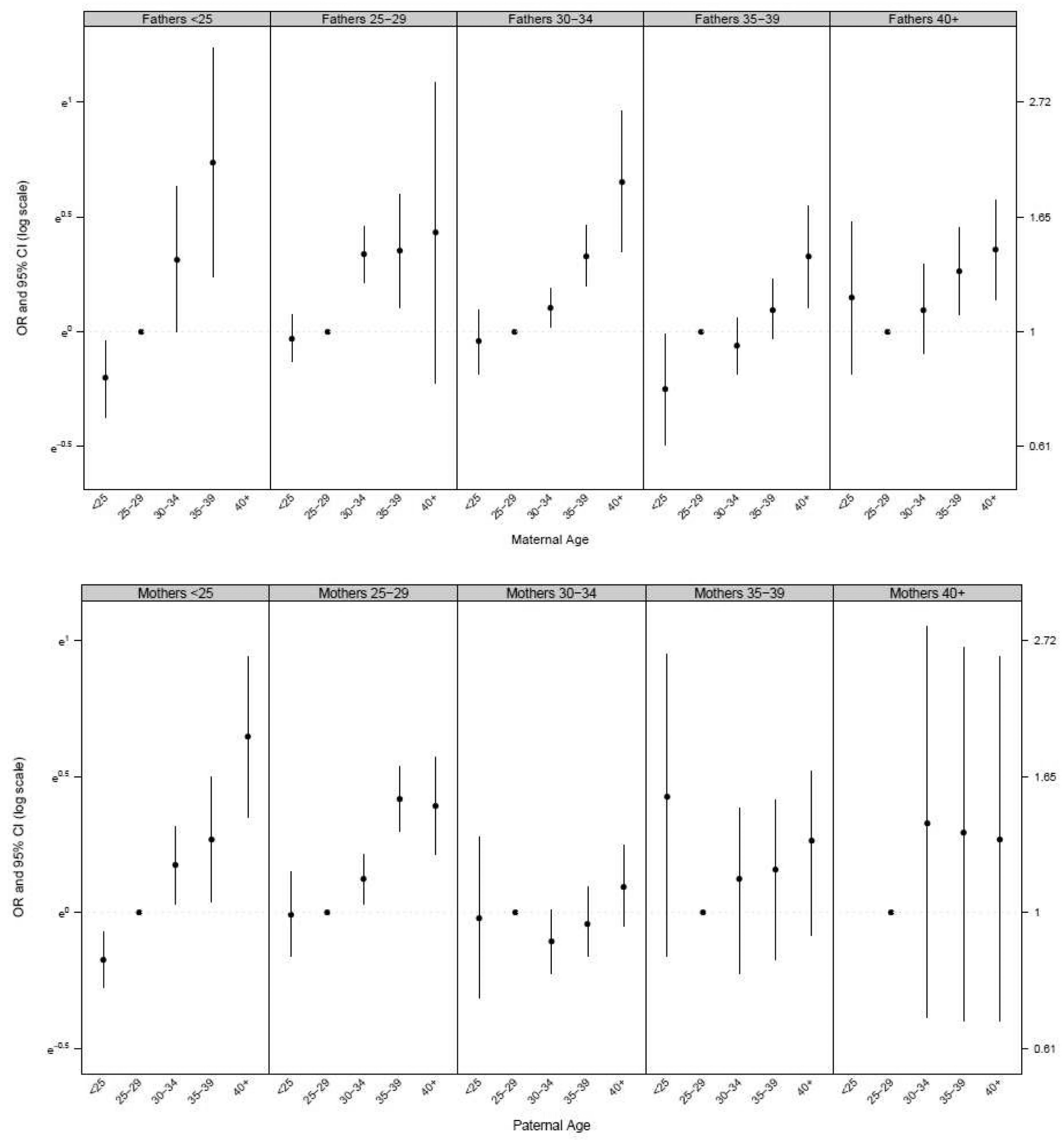

Figure 1.

Figure 1a. The effects of advancing maternal age within strata of paternal age, California births 1990-2002. Each panel shows the odds ratios and 95\% confidence intervals for increasing categories of maternal age within paternal age categories ranging from $<25$ years to $40+$ years. All odds ratios are adjusted for continuous age of restricted group, sum of 
parental education, maternal and paternal race/ethnicity, year of birth, parity, and insurance payment type. All confidence intervals include the variance inflation factor. All 95\% confidence intervals have been adjusted for the potential impact of multiple births from the same mother using a variance inflation factor, as described in the text. The minimum case $\mathrm{N}$ for any observation is 11 for the category of fathers 25-29 and mothers 40+.

Figure $1 \mathrm{~b}$. The effects of advancing paternal age within strata of maternal age, California births 1990-2002. Each panel shows the odds ratios and 95\% confidence intervals for increasing categories of paternal age within maternal age categories ranging from $<25$ years to $40+$ years. All odds ratios are adjusted for continuous age of restricted group, sum of parental education, maternal and paternal race/ethnicity, year of birth, parity, and insurance payment type. All confidence intervals include the variance inflation factor. All 95\% confidence intervals have been adjusted for the potential impact of multiple births from the same mother using a variance inflation factor, as described in the text. The category for mothers over 40 and fathers $<25$ was suppressed due to a case $\mathrm{N}$ of 3. 


\section{Table 1}

Demographics of study population, California births 1990-1999.

\begin{tabular}{|c|c|c|}
\hline & $\begin{array}{c}\text { Autism } \\
\mathbf{N}=12,159 \\
\mathbf{N}(\%)\end{array}$ & $\begin{array}{c}\text { All other births } \\
\mathbf{N}=4,935,776 \\
\mathbf{N}(\%)\end{array}$ \\
\hline Maternal age & Median $=30$ & Median $=27$ \\
\hline$<25$ & $2,689(22.1)$ & $1,713,971(34.7)$ \\
\hline $25-29$ & $3,304(27.2)$ & $1,406,234(28.5)$ \\
\hline $30-34$ & $3,576(29.4)$ & $1,161,890(23.5)$ \\
\hline $35-39$ & $2,089(17.2)$ & $541,102(11.0)$ \\
\hline 40 and up & $501(4.1)$ & $112,579(2.3)$ \\
\hline Paternal age & Median $=32$ & Median $=29$ \\
\hline$<25$ & $1,633(13.4)$ & $1,165,115(23.6)$ \\
\hline $25-29$ & $2,732(22.5)$ & $1,312,101(26.6)$ \\
\hline $30-34$ & $3,485(28.7)$ & $1,269,013(25.7)$ \\
\hline $35-39$ & $2,613(21.5)$ & $760,074(15.4)$ \\
\hline 40 and up & $1,696(14.0)$ & $429,473(8.7)$ \\
\hline \multicolumn{3}{|l|}{ Year of birth } \\
\hline 1990 & $679(5.6)$ & $539,325(10.9)$ \\
\hline 1991 & $832(6.8)$ & 535,477 (10.9) \\
\hline 1992 & $995(8.2)$ & $527,262(10.7)$ \\
\hline 1993 & $1,011(8.3)$ & $511,330(10.4)$ \\
\hline 1994 & $1,165(9.6)$ & $503,296(10.2)$ \\
\hline 1995 & $1,197(9.8)$ & $486,236(9.9)$ \\
\hline 1996 & $1,304(10.7)$ & $473,736(9.6)$ \\
\hline 1997 & $1,464(12.0)$ & $455,472(9.2)$ \\
\hline 1998 & $1,681(13.8)$ & $451,857(9.2)$ \\
\hline 1999 & $1,831(15.1)$ & $451,786(9.2)$ \\
\hline \multicolumn{3}{|l|}{ Sex of child } \\
\hline Female & 2,135 (17.6) & $2,525,301(51.2)$ \\
\hline Male & $10,024(82.4)$ & $2,410,443(48.8)$ \\
\hline Unknown & $0(0.0)$ & $6(0.0)$ \\
\hline \multirow{2}{*}{\multicolumn{3}{|c|}{$\begin{array}{l}\text { Years of combined } \\
\text { parental education }\end{array}$}} \\
\hline & & \\
\hline Less than 24 years & $2,340(19.3)$ & $1,774,323(36.0)$ \\
\hline 24 to 29 & $5,577(45.9)$ & $2,124,566(43.0)$ \\
\hline 30 years or more & $4,242(34.9)$ & $1,036,888(21.0)$ \\
\hline \multicolumn{3}{|l|}{ Mother's race/ethnicity } \\
\hline White & $5,241(43.1)$ & $1,877,954(38.0)$ \\
\hline Black & $986(8.1)$ & $335,455(6.8)$ \\
\hline Hispanic & $4,045(33.3)$ & $2,147,886(43.5)$ \\
\hline Asian & $1,697(14.0)$ & $484,573(9.8)$ \\
\hline Other & $174(1.4)$ & 80,055 (1.6) \\
\hline
\end{tabular}




\begin{tabular}{|rcc|}
\hline & $\begin{array}{c}\text { Autism } \\
\mathbf{N = 1 2 , 1 5 9} \\
\mathbf{N}(\%)\end{array}$ & $\begin{array}{c}\text { All other births } \\
\mathbf{N = 4 , 9 3 5 , 7 7 6} \\
\mathbf{N}(\mathbf{\%})\end{array}$ \\
\hline Missing & $16(0.2)$ & $9,853(0.2)$ \\
Father's race/ethnicity & & \\
Bhite & $5,518(45.4)$ & $1,851,553(37.5)$ \\
Black & $1,033(8.5)$ & $401,231(8.1)$ \\
Hispanic & $3,903(32.1)$ & $2,151,052(43.6)$ \\
Asian & $1,483(12.2)$ & $437,193(8.9)$ \\
Other & $200(1.6)$ & $83,796(1.7)$ \\
Missing & $22(0.2)$ & $10,951(0.2)$ \\
& & \\
Parity & & \\
Less than 2 & $9,633(79.2)$ & $3,469,888(70.3)$ \\
More than 2 & $2,526(20.8)$ & $1,465,890(29.7)$ \\
Insurance Payment & & \\
Type $=$ & & \\
Private & $8,652(71.2)$ & $2,761,317(55.9)$ \\
Public & $3,457(28.4)$ & $2,153,693(43.6)$ \\
Other & $50(0.41)$ & $20,767(0.42)$ \\
\hline & &
\end{tabular}

$=$ Insurance payment type was categorized as public if the anticipated form of payment was Medicare, medical, workers compensation, title $\mathrm{V}$, or other government programs. Private insurance type included Blue Cross/Blue Shield, private insurance company, health maintenance organization/ prepaid health plan, or self pay. Other insurance type included no charge, medically indigent, medically unattended birth or unknown. 


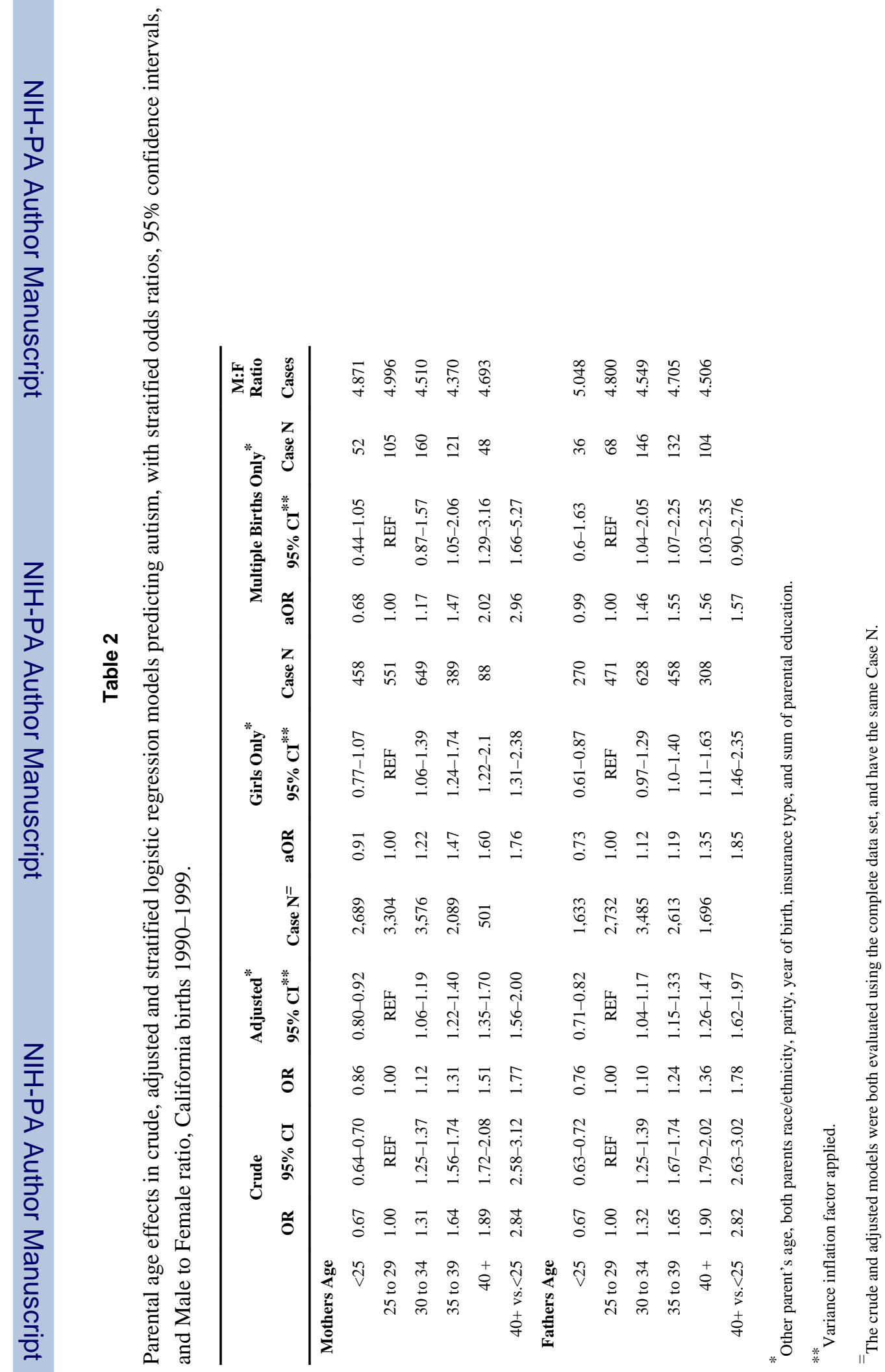




\section{Table 3}

The effect of paternal age among mothers less than 30 years old as compared to mothers equal to or over 30 . These results were computed by running the full model with an interaction term for the paternal age term (categorical) and a binary indicator for maternal age less than 30 and main effects to adjust for maternal and paternal age (categorical), maternal and paternal race/ethnicity, parity, year of birth, insurance type, and the sum of parental education. The confidence intervals include a variance inflation factor adjustment to account for the effects of multiple births from the same mother, as described in the text.

\begin{tabular}{|c|c|c|c|c|}
\hline & \multicolumn{2}{|l|}{ Mother $<30$} & \multicolumn{2}{|c|}{ Mother $=$ or $>30$} \\
\hline & aOR & $95 \% \mathrm{CI}^{*}$ & aOR & $95 \% \mathrm{CI}^{*}$ \\
\hline Father $<25$ & 0.76 & $0.70-0.82$ & 1.11 & $0.86-1.42$ \\
\hline $25-29$ & 1 & REF & 1.00 & REF \\
\hline $30-34$ & 1.15 & $1.07-1.24$ & 0.94 & $0.85-1.05$ \\
\hline $35-39$ & 1.49 & $1.35-1.66$ & 1.01 & $0.91-1.13$ \\
\hline Father $>40$ & 1.59 & $1.37-1.85$ & 1.13 & $1.01-1.27$ \\
\hline
\end{tabular}


Table 4

Comparison of the actual number of cases of autism among children born in California in 1990 to what would be projected had the age breakdown of mothers then been the same as they were in 1999 .

\begin{tabular}{|c|c|c|c|c|c|c|c|}
\hline & \multicolumn{2}{|c|}{1990 Births } & \multirow{2}{*}{$\begin{array}{c}H \\
1999 \\
\text { Maternal } \\
\text { Age } \\
\text { Distribution } \\
(\%)\end{array}$} & \multicolumn{3}{|c|}{$\begin{array}{l}\text { Autism Cases Diagnosed Prior to Age } 6 \text { among } \\
\text { children born in } 1990\end{array}$} & \multirow[b]{2}{*}{$P-A$} \\
\hline $\begin{array}{l}\text { Mothers } \\
\text { Age }\end{array}$ & $\#$ & $\begin{array}{c}\text { Maternal } \\
\text { Age } \\
\text { Distribution } \\
(\%)\end{array}$ & & $\begin{array}{c}A \\
\text { Actual \# }\end{array}$ & $\begin{array}{c}R \\
\text { Rate } \\
\text { (per 10,000 births) }\end{array}$ & $\underset{\#}{P^{I}}$ & \\
\hline LT 20 & 70,964 & 11.6 & 11.1 & 58 & 8.2 & 55.8 & -2.2 \\
\hline 20-24 & 159,839 & 26.1 & 23.2 & 186 & 11.6 & 164.9 & -21.1 \\
\hline $25-29$ & 183,410 & 29.9 & 26.6 & 281 & 15.3 & 249.4 & -31.6 \\
\hline 30-34 & 133,762 & 21.8 & 23.5 & 305 & 22.8 & 328.3 & 23.3 \\
\hline 35-39 & 54,609 & 8.9 & 12.7 & 135 & 24.7 & 192.2 & 57.2 \\
\hline GE 40 & 10,219 & 1.7 & 2.9 & 27 & 26.4 & 46.9 & 19.9 \\
\hline Total & 612,803 & 100 & 100 & 992 & & 1037.6 & $45.6^{2}$ \\
\hline
\end{tabular}

(1) The projected number of cases is computed by applying the 1990 autism case rates $(R)$ to the hypothetical number of births that would have occurred in 1990 in each row had the total number of 1990 births $(612,803)$ been distributed as in $1999(H): \mathrm{P}=(612,803 \times \mathrm{H} \times \mathrm{R})$.

${ }^{2}$ This represents the projected number of additional cases of autism that would have occurred among children born in 1990 had the maternal age distribution then been the same as in 1999 